\title{
Analysis of Human-Robot Spatial Behaviour applying a Qualitative Trajectory Calculus
}

\author{
Marc Hanheide $^{1}$, Annika Peters ${ }^{2}$, and Nicola Bellotto ${ }^{1}$
}

\begin{abstract}
The analysis and understanding of human-robot joint spatial behaviour (JSB) - such as guiding, approaching, departing, or coordinating movements in narrow spaces - and its communicative and dynamic aspects are key requirements on the road towards more intuitive interaction, safe encounter, and appealing living with mobile robots. This endeavours demand for appropriate models and methodologies to represent JSB and facilitate its analysis. In this paper, we adopt a qualitative trajectory calculus (QTC) as a formal foundation for the analysis and representation of such spatial behaviour of a human and a robot based on a compact encoding of the relative trajectories of two interacting agents in a sequential model. We present this QTC together with a distance measure and a probabilistic behaviour model and outline its usage in an actual JSB study. We argue that the proposed QTC coding scheme and derived methodologies for analysis and modelling are flexible and extensible to be adapted for a variety of other scenarios and studies.
\end{abstract}

\section{INTRODUCTION}

At an increasing pace mobile robots enter our everyday environments. They begin to share living spaces with us and start becoming useful companions and assistants. In such scenarios, from a roboticist's point of view, the ability to move about and safely manoeuvre in human-populated spaces is a key aspect for these mobile robots [1]. A large body of research is thus dedicated to provide answers to questions on joint spatial behaviour and spatial management in Human-Robot Interaction (HRI). In early works, humans were merely seen as static obstacles [2] that had to be avoided by a robot. Then, their dynamic aspects have been taken into account (e.g. [3]). In more recent works, robots explicitly plan to move on more "socially acceptable and legible paths" [4], [5], [6]. The term "legible" here refers to the communicative - or interactive - aspects of motions which previously has widely been ignored in robotics research. Looking at spatial behaviour of humans, [7] explain that humans also have to consider the actions of others as well, when planning their own actions. Hence, moving around is also about communication and coordination of one's movements - at least in cases where people are walking within a certain vicinity to one another, e.g. entering each other's personal or social spaces [8]. Expressed in Watzlawick's famous words "One cannot not communicate" [9], any motion of the robot will incite a communicative

\footnotetext{
${ }^{1}$ M. Hanheide and N. Bellotto are with the School of Computer Science, University of Lincoln, Lincoln, LN6 7TS, England, \{mhanheide, nbellotto\}@lincoln.ac.uk

${ }^{2} \mathrm{~A}$. Peters is with the Center of Excellence "Cognitive Interaction Technology" (CITEC), Bielefeld University, Universitätsstraße 21-23, 33615 Bielefeld, Germany, apeters@techfak.uni-bielefeld.de
}

interpretation in humans who observe this motion. For more intuitive, and consequently usually also more efficient, spatial management, we have to gain a better understanding of joint spatial behaviour and its communicative aspects. This paper aims to contribute to this better understanding by proposing a novel coding scheme of dynamic spatial relations of two interacting, moving agents; with a particular focus of its potential for human-robot interaction studies and spatial behaviour modeling.

Communication and interpretation by humans usually takes place in qualitatively represented domains. For instance, a human will simply refer to another person standing next to her as "to my right", even though the actual angle between her own facing direction and the imaginary line between the two persons actually is meetings at an angle of, e.g., $70.4^{\circ}$. Hence, qualitative abstractions of the actual quantities appear adequate to facilitate understanding and comparison of spatial behaviour. Qualitative abstraction is therefore also a core concept of the proposed coding scheme which is based on a variant of the Qualitative Trajectory Calculus (QTC). QTC has first been put forward by Van de Weghe et al. as "a language for representing and reasoning about movements of objects in a qualitative framework" [10]. It allows for a very compact representation of the dynamic trajectories of two agents, and - as a calculus - provides a sound mathematical foundation with well-defined rules for composition and qualitative reasoning. The variant $\mathrm{QTC}_{C}$ we chose as the basis for our work here allows to encode relative motion on a $2 \mathrm{D}$ plane in sequences of finite states as will be detailed in Sec II. Based on this coding scheme we propose a metric to compare different traces of joint spatial behaviour of two interacting agents (here, a human and a robot). This allows to (i) quantitatively compare different trials, and (ii) to derive clusters of similar behaviour. Furthermore, we can look at traces of joint spatial behaviour in a probabilistic sequential framework that allows to infer most probable models of behaviour in specific situations. In Sec. IV we will present these ideas for QTC-based analysis and modelling in some detail after having introduced the case study on joint spatial behaviour in Sec. III to underpin our contribution. Finally, we will conclude this paper with a critical assessment of the proposed coding scheme and outline possible extensions.

\section{Qualitative Trajectory Calculus For two Agents MOVIng IN 2D}

The qualitative representation of human motion behaviours, like typical walking paths and dynamic trajectories, 


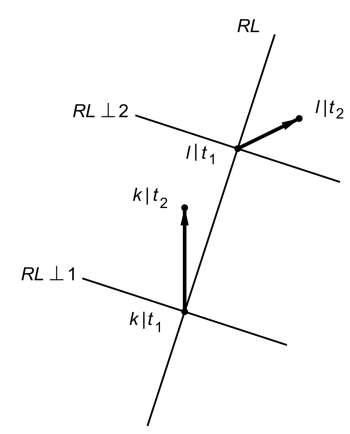

Fig. 1. The QTC Double Cross (reproduced from [10]).

depends on the subjective interpretation of who describes them, and is therefore very difficult to encode in computer languages. On the other hand, the use of qualitative spatial terms is a powerful method to abstract a wide number of possible HRI's scenarios, maybe conceptually similar but numerically (in terms of kinematic properties) very different. This can be extremely useful to analyse human-robot spatial interactions for further reasoning and for implementing robot's behaviours that are more socially acceptable.

A compact representation of the spatial relations between two moving points has been proposed by Van de Weghe [11] with the Qualitative Trajectory Calculus. QTC is a mathematical formalism that belongs to the broader area of qualitative spatial representation and reasoning [12], from which it inherits several properties and computational tools. It has been recently shown in [13] that QTC can be used to represent simple human-robot spatial interactions, like approach behaviours, and trigger opportune robot control strategies. Differently from that work, in the current research we adopt a variant of the calculus, called QTC Double Cross $\left(\mathrm{QTC}_{C}\right)$, that considers also the direction of the moving agents. QTC $C_{C}$ is a necessary first step towards representing more complex human-robot interactions, in particular when the distance between the two agents is relatively small.

$\mathrm{QTC}_{C}$ represents the relative motion of two points, $k$ and $l$, with respect to the reference line $R L$ that connects them, as shown in Fig. 1. We use a simple version of $\mathrm{QTC}_{C}$ that considers only the distance and the relative direction of each point with respect to the other (see [14] for an introduction to different variants of QTC). In this case, four qualitative relations are defined as follows:

1) movement of $k$ with respect to $l$ at time $t$

- $: k$ is moving towards $l$

$0: k$ is neither moving towards to nor away from $l$

$+: k$ is moving away from $l$

2) movement of $l$ with respect to $k$ at time $t$

- same as 1), but with $k$ and $l$ swapped

3) movement of $k$ with respect to $R L$ at time $t$

- $: k$ is moving to the left-hand side of $R L$

$0: k$ is moving along $R L$ or not moving at all

$+: k$ is moving to the right-hand side of $R L$

4) movement of $l$ with respect to $R L$ at time $t$

- same as 3), but with $k$ substituted by $l$

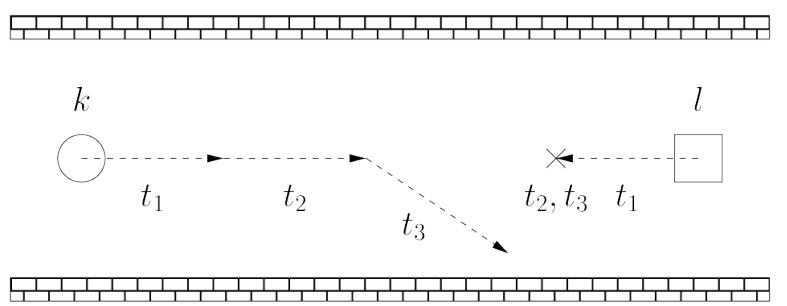

Fig. 2. Example of spatial interaction where the robot (agent $l$ ) stops to let the person (agent $k$ ) pass on its left.

The relative motion of two points at time $t$, therefore, can be expressed by a 4-elements state descriptor such as $(-+0-)_{t}$, which in this case means " $k$ moves towards $l$; $l$ moves away from $k ; k$ moves along $R L ; l$ moves on the left-hand side of $R L$ ".

The total number of possible states is therefore $3^{4}=81$. Combined in temporal sequences, they can be used to represent different scenarios of spatial interaction between two agents. Consider the following example, illustrated also in Fig. 2: A person and a robot move towards each other along a narrow corridor; when close enough, the robot stops to let the person pass on its left-hand side. If $k$ is the person and $l$ the robot, this situation can be described in $\mathrm{QTC}_{C}$ by the following temporal sequence:

$$
\left(\begin{array}{lll}
- & 0 & 0
\end{array}\right)_{t_{1}} \rightsquigarrow\left(\begin{array}{lll}
-0 & 0 & 0
\end{array}\right)_{t_{2}} \rightsquigarrow\left(\begin{array}{ll}
-0 & 0
\end{array}\right)_{t_{3}}
$$

QTC $_{C}$ has been used in other complex motion behaviours to analyse traffic situations and sport events [14]. In all those cases, and in the one discussed next, the spatial relations between the observed targets have been visually annotated under expert supervision. However, if the human position is independently observed by a robot through its sensors (e.g. a laser scanner), then the relative measures tend to be less accurate with the increase of the distance between robot and human, which in turn could corrupt the QTC states. One way to tackle this problem is to use a probabilistic model, as proposed in Sec. IV-B, including the uncertainty of the QTC state transitions. Another solution would be also to "switch" between different QTC representations (e.g. QTC ${ }_{B}$ for large distances, $\mathrm{QTC}_{C}$ for short ones - see [11] for a full description of QTC variants) in an automatic fashion and according to the desired interaction behaviour. This is left for future extensions of our research.

\section{A CAse Study: PASSING IN A NARROW CorRidor}

We have first applied the methodology proposed in this paper in an analysis of Human-Robot spatial behaviour concerned with spatial management in narrow spaces, namely a human and robot passing in a narrow corridor setting. This analysis shall serve as a vehicle for a more detailed explanation of the method itself and also indicate its applicability in HRI analysis. Consider a situation as illustrated in Fig 3(a) (taken from our actual study) where a human encounters a mobile robot driving towards her in a rather narrow passageway or corridor. In our study, we were interested in the joint spatial behaviour under different pre-programmed conditions of robot behaviour. For this paper, we will not 


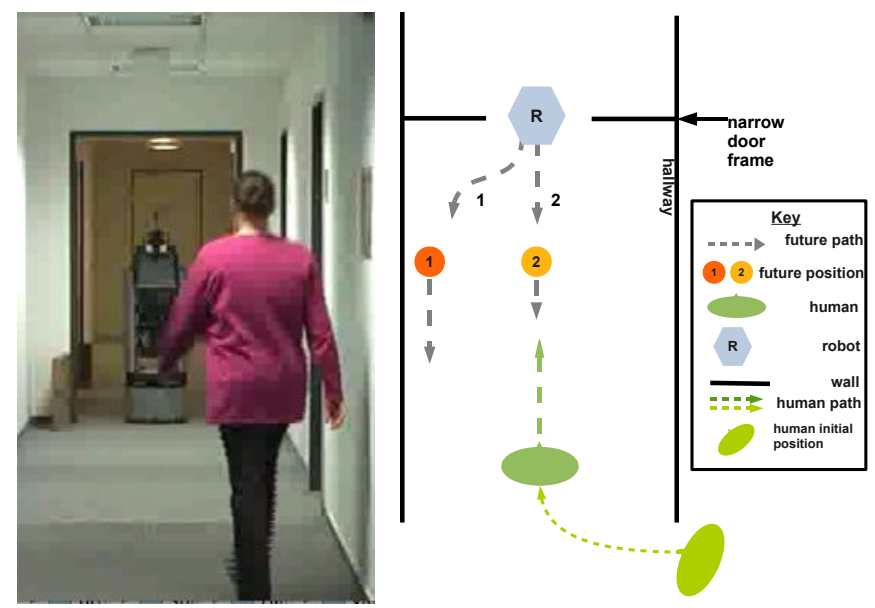

(a) An uninstructed subject (b) The two different conditions of prespontaneously encountering a programmed robot behaviour. mobile robot in the corridor.

Fig. 3. The corridor study on joint spatial behaviour.

present the whole study in detail but focus on two conditions (of a total of eight in the original study) to highlight aspects of our analysis. Further details regarding the study can be found in [15] and [16].

\section{A. Robot Platform}

The study was carried out with the robot platform "BIRON" at the University of Bielefeld. BIRON is a combination of a two-wheeled PatrolBot and GuiaBot manufactured by Adept MobileRobots shown in Fig. 3(a). The robot comprises a laser range finder (SICK LMS200) which covers $180^{\circ}$ in front of the robot at about $30 \mathrm{~cm}$ above the floor. The data of the laser range finder is used to build a map of the environment and form hypotheses of humans legs in the robots vicinity. The camera is mounted on the top of the robot to record video data for later analysis and simultaneously detect faces of humans. Besides two powered wheels BIRON has two rear casters to maintain its balance. BIRON has an overall size of approximately $0.5 m(w) \times$ $0.6 m(\mathrm{~d}) \times 1.3 m(\mathrm{~h})$. For this study the robot was running a stripped-down version of the software system also used at the RobotCup@HOME competition [17] in 2010, enabling the robot to autonomously plan paths, avoid obstacles, and detect and track humans in its vicinity.

\section{B. Procedures \& Conditions}

The study was implemented as an observational case study to investigate spontaneous and non-primed behaviour. The robot was positioned in the corridor depicted in Fig 3(a) ready to autonomously drive towards an approaching person. The corridor is $1.85 \mathrm{~m}$ wide with a narrow door opening of $1.15 \mathrm{~m}$ at the starting position of the robot (cf. Fig. 3(b)).

A total of 59 participants ( $34 \%$ female, $66 \%$ male, age average $M=30.4$, standard deviation $S D=7.6$ ) were recruited on the Bielefeld campus, Germany, to participate in a robot interaction study. Most participants have a computer science background (70.4\%), 17.9\% have another scientific background and $10.7 \%$ have no scientific background. To
TABLE I

ORIGINAL CODING SCHEME

\begin{tabular}{|c|c|}
\hline Variable & Categories \\
\hline $\begin{array}{l}\text { position } \\
\text { orientation }\end{array}$ & $\begin{array}{l}\text { \{left, half-left, middle, half-right, right }\} \\
\text { \{straight-facing, diagonally-facing, straight-averted, } \\
\text { diagonally-averted }\end{array}$ \\
\hline veloc & $\{$ slow, normal, fast $\}$ \\
\hline
\end{tabular}

test spontaneous behaviour, participants were welcomed by an experimenter before they could see the actual robot and deceived into believing they should go to a room at the other end of a corridor to participate in the actual study. They were not told that they will encounter a robot on their way. The robot's data (position, person tracking, on-board camera, etc.) were recorded and combined with a video captured by an external, hidden camera from a position behind the participants (cf. Fig. 3(a)). Participants took part in the study, unaware of the details and were only informed right after their encounter with the robot.

In the full study, eight different conditions were implemented as behaviours on the robot. These correspond to eight different goal positions for the robot, resulting in different motion patterns. These different patterns represent different levels of resoluteness of the robot to go along its way, i.e. the extremes are on the one hand a behaviour where the robot remain in the center of the corridor driving straight at the human (as also shown as condition 2 in Fig. 3(b)), and on the other a behaviour of backing off from the approaching human. The autonomous behaviour was started when the person became visible to the robot, i.e. came around the corner to enter the corridor.

In the main studies [15], [16] we were interested in the communicative appraisal of these motion patterns by the participants, assessed also through an accompanying questionnaire to be filled after they met the robot. In this paper, however, we focus only on the observed behaviour in two of the eight conditions, both shown in Fig. 3(b). Condition 1 is designed to be a rather polite behaviour, where the robot first drives through the doorway but then moves to its right-hand side to give way to the approaching subject. On the contrary, condition 2 is the above mentioned resolute behaviour where the robot will remain in the centre of the corridor and only reactively avoid the human if she is coming too close.

Of our 59 subjects encountering the robot, 5 were randomly selected to be exposed to condition 1 and 6 to condition 2 (The other subjects were exposed to one of the remaining 6 other condition not discussed in this paper). The motions of both, the subject and the robot, were annotated in ELAN ${ }^{1}$ by two coders independently following an agreed coding scheme denoting position within the corridor, orientation in relation to one another, and absolute velocity in qualitative categories shown in Tab. I. This original coding scheme was then automatically translated into simplified QTC $_{C}$ using an adapted version of the SALEM toolbox [18]. In order to do this, the ELAN annotation for each trial are

${ }^{1}$ http://www.lat-mpi.eu/tools/elan/ 


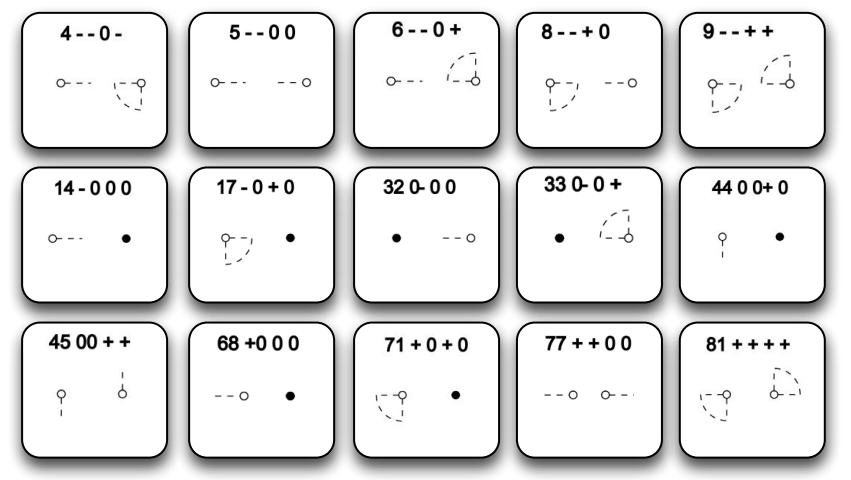

Fig. 4. All QTC ${ }_{C}$ states occurring in the trials. Illustration of the individual states reproduced from [10]. The left point in the illustrations of the states always represents the human, the right one the robot. For instance, state number " 5 " represents human and robot approaching straight to one another (corresponding to Fig. 3(a)), while state " 6 " indicates a human walking straight towards the robot, with the robot heading more to the right (from its own perspective) but still decreasing the distance to the human. More details on the notation of $\mathrm{QTC}_{C}$ are given in Sec. II.

unified into a sequence of $\mathrm{QTC}_{C}$ state descriptors, denoting the human as $k$ and the robot as $l$ in the definition given in Sec. II. The sequences were then trimmed to start when the human turned towards the robot after entering the corridor and end when the human passed the door frame indicated in Fig. 3(b). This annotation yields sequences of length varying between 5 and 8 QTC $_{C}$ states per trial, with 15 of the possible 81 QTC $_{C}$ states, shown in Fig. 4, actually occurring in those sequences.

\section{QTC-BASED ANALYSIS AND ModELling}

As stated in the previous section, the foundation for a QTC $_{C}$-based analysis is an annotation of the joint spatial behaviour of both the human and the robot in $\mathrm{QTC}_{C}$ state sequences. We have shown in the previous section how a "story" can be told as such a sequence and will now look at ways to facilitate analysis using this coding scheme. Obviously, the coding scheme allows to use any kind of analysis of sequences composed of finite states to be applied. In the following, we outline two methods that proved useful in our domain and we believe yield a more general applicability to the analysis of joint spatial behaviour. They serve two general objectives in experimental analysis: To compare different outcomes and to cluster similar ones, and to generalise into a - here probabilistic - model. The proposed methods are generally applicable to any kind of QTC-annotated behaviour, however, we will present use cases based on the corridor scenario for explanation and highlight some findings we made in this setting.

\section{A. The QTC Sequence Edit Distance (QSED)}

In order to compare different instances of behaviour encoded as $\mathrm{QTC}_{C}$ sequences, a distance metric is required. It forms the basis for any kind of similarity-based analysis like clustering or sample-based classification.

1) Definition: Let $\Sigma$ be the set of all 81 possible QTC $_{C}$ states plus a start and an end state $S$ and $E$. We can then define a QTC state sequences $T^{m}$ of length $N^{m}$ each as

$$
T^{m}=\left(\sigma_{1}^{m}, \sigma_{2}^{m}, \ldots, \sigma_{N^{m}}^{m}\right), \text { with } \sigma_{i}^{m} \in \Sigma .
$$

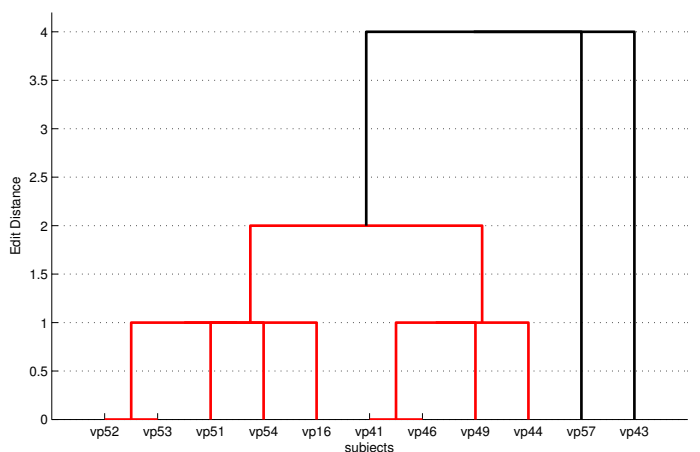

Fig. 5. A dendrogram of single-linkage agglomerative clustering using the QTC sequence edit distance (QSED).

In a $\mathrm{QTC}_{C}$ sequence, we guarantee that the same state cannot occur multiple times in direct sequence order, i.e. $\sigma_{i}^{m} \neq$ $\sigma_{i-1}^{m}$, by collapsing such duplications into a single one. With this definition, we can define the $\mathrm{QTC}_{C}$ Sequence Edit Distance (QSED) $Q:\left(T^{m}, T^{l}\right) \rightarrow l\left(t\left(T^{m}\right), t\left(T^{l}\right)\right)$ between two QTC $_{C}$ sequences with $l(\cdot, \cdot)$ being the Levenshtein distance defined on strings [19]. $t(\cdot)$ maps the $\mathrm{QTC}_{C}$ state sequence into corresponding string so that the Levenshtein distance function $l(\cdot, \cdot)$ can be applied. Consequently $Q$ computes the minimum number of edits required to transform one QTC sequence $T^{m}$ (equivalent to a string in the Levenshtein distance) into the other $T^{l}$, with the equally expensive edit operations, being insertion, deletion, or substitution of a single $\mathrm{QTC}_{C}$ state.

2) Distance-based Cluster Analysis in the Corridor Study: Being now able to compare different $\mathrm{QTC}_{C}$ sequences we can cluster them using agglomerative clustering methods. Here, we applied single linkage clustering based on the QSED between different sequences. The result of our cluster analysis for all trials conducted in the two conditions is shown as a dendrogram in Fig 5. On the horizontal axis the different trials are indicated with the anonymised subject identifier starting with 'vp'. The trials $T_{1}=\{v p 41, v p 43, v p 44, v p 46, v p 49\}$ have been carried out under condition 1 , while $T_{2}=$ $\{v p 16, v p 51, v p 52, v p 53, v p 54, v p 57\}$ are the trials under condition 2. The ordinate denotes the minimal QSED between the respective clusters.

It can be seen that the trials $v p 16, v p 51, v p 52, v p 53$, and $v p 54$ are very similar. They all belong to condition 2 and we can see that they only have a maximum QSED of 1 between them. The same is true for $v p 41, v p 44, v p 46$, and $v p 49$, which all belong to condition 1. So generally, the conditions form well-defined clusters. However, we can see two outliers $v p 43$ and $v p 57$ which significantly deviate from the others. Taking a closer look at those two trials it becomes apparent that they indeed represent outliers in behaviour. In $v p 43$ the robot stopped its autonomous motion, likely due to some detected obstacle, and then only later on continued its journey down the corridor. Hence it incurred more state changes in the $\mathrm{QTC}_{C}$ sequence. In $v p 57$ on the 
other hand, we can see a kind hesitation gesture of the human. Here the human first approaches the robot, and then stops (state 32 in Fig. 4), unsure how to proceed with the robot driving straight at her. Then, after this hesitation, the subject continues her way by passing the robot on its lefthand side. Interestingly, all participants passed the robot on this side even in condition 2 where the robot remained in the centre of the corridor, indicated by the absence of any QTC $_{C}$ states with "-" in the third component. We deem this to be due to a cultural preference of right-hand side traffic in Germany but have not tested this hypothesis any further yet.

\section{B. A Probabilistic Model of Joint Spatial Behaviour}

Complementing comparison of different behaviour traces, also the analysis of $\mathrm{QTC}_{C}$ state sequences using a probabilistic framework yields useful findings to the understanding and modelling of joint spatial behaviours. As we have a finite set $\Sigma$ of QTC ${ }_{C}$ states and observed transitions between them through actual experiments, we can encode a certain class of behaviour using a Markov model [20]. Note, that here we modelled it as a Markov chain as we can indeed observe the underlying states directly. It is a natural extension of this work to employ Hidden Markov Models (HMM) to account for uncertainty in the actual annotation and recognition process. This makes the model applicable, for instance, in automatic behaviour recognition or to control a robot in more compliant ways based on the observed pattern as proposed in [21]. Fig. 6 depicts the two Markov chains that have been estimated from the actually observed states and transition frequencies in the two conditions of our study. QTC $_{C}$ states are represented as ellipses. The state numbers correspond to the $\mathrm{QTC}_{C}$ state definitions given in Fig. 4. The edges between nodes represent transitions that occurred annotated with the estimated probability of their occurrence which also is reflected by the width of the respective edge's line. It becomes apparent that certain transitions are clearly dominating while others are rare. Given the transition probabilities, we can now compute the most probable path from the start state $S$ to the end state $E$ in the respective models for both conditions. For condition 1 this sequence is

$$
\hat{T}_{1}=S \rightsquigarrow 17 \rightsquigarrow 14 \rightsquigarrow 5 \rightsquigarrow 6 \rightsquigarrow 9 \rightsquigarrow 45 \rightsquigarrow 81 \rightsquigarrow E
$$

with an overall product probability of 0.118 . For condition 2 the most probable sequence is

$$
\hat{T}_{2}=S \rightsquigarrow 5 \rightsquigarrow 8 \rightsquigarrow 9 \rightsquigarrow 45 \rightsquigarrow 81 \rightsquigarrow E
$$

with a production probability of 0.1389 . This sequence is also depicted in Fig. 7 with an illustration of the actual QTC ${ }_{C}$ states involved.

Two main observation can be made: First, the tails of the two most probable sequences are the same $(9 \rightsquigarrow 45 \rightsquigarrow 81 \rightsquigarrow E$ ), indicating that the actual passing is very similar. But before this part, we can see $5 \rightsquigarrow 6 \rightsquigarrow 9$ in condition 1 while in condition 2 we observe $5 \rightsquigarrow 8 \rightsquigarrow 9$ as the most probable sequence. Looking back at Fig. 4 it becomes clear that state number 6 which occurs in condition

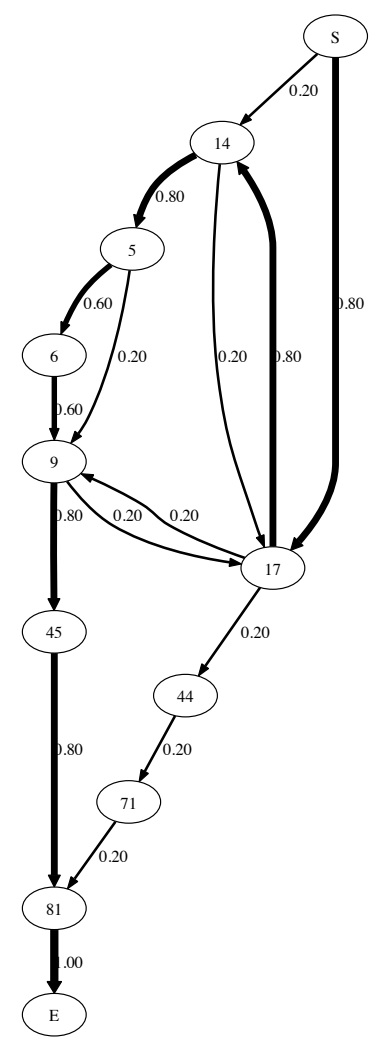

(a) Condition 1 .

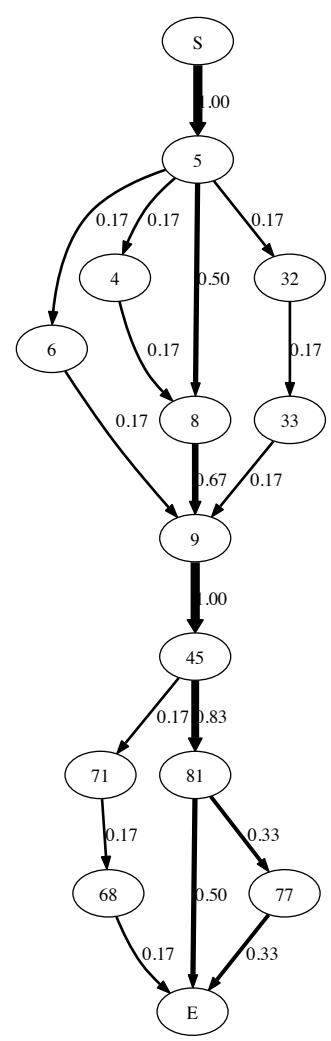

(b) Condition 2 .
Fig. 6. Markov chain model of the two conditions with transition probabilities between $\mathrm{QTC}_{C}$ states. Please refer to Fig. 4 for an explanation of the state numbers.

1 corresponds to the robot first driving to the side, i.e communicating to the subject that it will make room, while state number 8 , occurring in condition 2 only, represents the subject first making an effort to avoid getting too close to the robot. So the implemented spatial behaviour clearly is reflected in the probabilistic model.

Second, it can be observed that in the majority of the cases in condition 1 the robot was not yet moving when the subject entered the corridor, reflected by the state sequence $(S \rightsquigarrow 17 \rightsquigarrow 14)$ at the beginning of $\hat{T}_{1}$. This is due to the design of the experiments in this setting.

\section{Discussion \& CONCLUSION}

We have proposed a novel formalism to represent and analyse joint spatial behaviour in human-robot interaction. The key contribution of our work is the adoption of a qualitative trajectory calculus $\left(\mathrm{QTC}_{C}\right)$ for this domain and a set of methods to compare and to model behaviour based on $\mathrm{QTC}_{C}$ sequences. We have presented this novel methodology employed in study of joint spatial behaviour in a passing-by scenario involving a human and robot and were able to show the applicability of our method in such a domain. Adopting $\mathrm{QTC}_{C}$ gave us the benefit of qualitative abstraction together with a sound formal foundation and well defined states. It can be automatically generated from existing annotation as done in this work or directly be employed as a coding scheme in HRI spatial behaviour studies. 


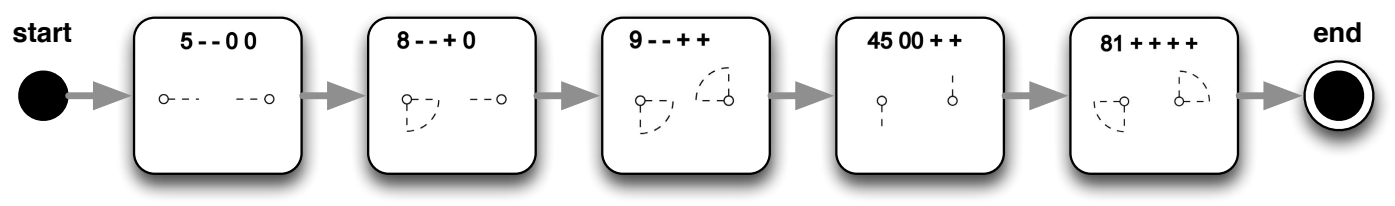

Fig. 7. Most probable state sequence in condition 2 (production probability $P\left(\hat{T}^{2}\right)=0.1389$ ).

Certainly there are various ways to extend and improve these initial ideas and concepts. First of all, many other techniques to compare and classify sequences of discrete states can be applied, like HMM, dynamic time warping (to take the timing into account), and others more. Also, our distance measure QSED has the potential to be employed in variety classification and recognition algorithms that are based on distances. We have yet to explore the full potential of these opportunities also in other domains and studies. Also, we like to actually employ the analysis of behaviour online in order to effect the robot's behaviour based on the behaviour of the human. Some initial work [13] on the topic also using a simpler version of QTC proved promising.

For the analysis of actual human-robot joint behaviours we are convinced that further aspects need to be taken into consideration and demand an extension of the currently used calculus. So it is well-known that distances between a robot and a human play an important role in spatial management [8], [22]. However those are currently not represented. Also body orientation and posture are neglected so far, rendering $\mathrm{QTC}_{C}$ well suitable for quite a few, but certainly not all classes of studies on spatial behaviour.

With regard to the use of $\mathrm{QTC}_{C}$, we have not taken opportunity of various of its beneficial features. For instance, whether $\mathrm{QTC}_{C}$ define restrictions on possible transitions between states in so-called conceptual neighbourhood diagrams. Not all transitions between states are directly allowed in $\mathrm{QTC}_{C}$ as they will inevitably pass through one or several intermediate states [10]. These restrictions should be taken into account when building models and could also be factored into QSED to assign lower costs to substitutions of conceptually neighbouring states. Furthermore, we will be looking at ways to combine different variants of QTC (going beyond the $\mathrm{QTC}_{C}$ variant employed here) to yield a more powerful and versatile coding scheme to spatial behaviour analysis and generation.

\section{ACKNOWLEDGMENT}

This work has partially been funded by the German Research Foundation (DFG) under the contracts of the Cluster of Excellence "Cognitive Interaction Technology" (CITEC ${ }^{2}$, EXC 277).

\section{REFERENCES}

[1] A. Steinfeld, T. Fong, D. Kaber, M. Lewis, J. Scholtz, A. C. Schultz, and M. Goodrich, "Common metrics for human-robot interaction," Proceeding of the 1st ACM SIGCHI/SIGART conference on Humanrobot interaction - HRI '06, p. 33, 2006.

[2] J. Borenstein and Y. Koren, "Real-time obstacle avoidance for fact mobile robots," IEEE Transactions on Systems, Man and Cybernetics, vol. 19, no. 5, pp. 1179-1187, 1989.

${ }^{2}$ https://www.cit-ec.de/research/SPACON
[3] R. Simmons, "The curvature-velocity method for local obstacle avoidance," in IEEE International Conference on Robotics and Automation, vol. 4, no. April. Minneapolis, Minnesota: IEEE, 1996, pp. 33753382.

[4] E. Sisbot, L. Marin-Urias, R. Alami, and T. Simeon, "A Human Aware Mobile Robot Motion Planner," IEEE Transactions on Robotics, vol. 23, no. 5, pp. 874-883, Oct. 2007.

[5] M. Yoda and Y. Shiota, "Analysis of human avoidance motion for application to robot," in Proceedings 5th IEEE International Workshop on Robot and Human Communication. RO-MAN'96 TSUKUBA. IEEE, 1996, Conference proceedings (whole), pp. 65-70.

[6] D. J. Feil-Seifer and M. J. Matarić, "People-Aware Navigation For Goal-Oriented Behavior Involving a Human Partner," in Proceedings of the International Conference on Development and Learning, Frankfurt am Main, Germany, Aug. 2011.

[7] T. Ducourant, S. Vieilledent, Y. Kerlirzin, and A. Berthoz, "Timing and distance characteristics of interpersonal coordination during locomotion," Neuroscience Letters, vol. 389, no. 1, pp. 6-11, November 2005.

[8] E. T. Hall, "Proxemics," Current Anthropology, vol. 9, no. 2/3, pp. pp. 83-108, 1968.

[9] P. Watzlawick, J. B. Bavelas, and D. D. Jackson, Pragmatics of human communication - a study of interactional patterns, pathologies, and paradoxes. New York: Norton, 1967.

[10] N. Van de Weghe, B. Kuijpers, and P. Bogaert, "A Qualitative Trajectory Calculus and the Composition of its Relations," GeoSpatial, vol. 281, pp. 60-76, 2005.

[11] N. Van de Weghe, "Representing and reasoning about moving objects: A qualitative approach," Ph.D. dissertation, Ghent University, 2004.

[12] A. G. Cohn and J. Renz, "Chapter 13 Qualitative Spatial Representation and Reasoning," in Handbook of Knowledge Representation, F. van Harmelen, V. Lifschitz, and B. Porter, Eds. Elsevier, 2008, vol. 3, pp. 551-596.

[13] N. Bellotto, "Robot control based on qualitative representation of human trajectories," in AAAI Spring Symposium - Designing Intelligent Robots: Reintegrating AI, Stanford, CA, USA, 26-28 March 2012, tR SS-12-02.

[14] M. Delafontaine, "Modelling and analysing moving objects and travelling subjects: Bridging theory and practice," Ph.D. dissertation, Department of Geography, Ghent University, 2011.

[15] A. Peters, T. P. Spexard, P. Weiß, and M. Hanheide, "Make room for me - A spatial and situational movement concept in HRI," in Workshop on Behavior Monitoring and Interpretation, Paderborn, Germany, 2009.

[16] A. Peters, T. P. Spexard, M. Hanheide, and P. Weiss, "Hey robot, get out of my way - A survey on a spatial and situational movement concept in HRI," in Behaviour Monitoring and Interpretation - BMI - Well-Being, 2011, pp. 147-165.

[17] S. Wachsmuth, F. Siepmann, D. Schulze, and A. Swadzba, "ToBI - Team of Bielefeld: The Human-Robot Interaction System for RoboCup@Home 2010,”2010.

[18] M. Hanheide, M. Lohse, and A. Dierker, "SALEM - Statistical AnaLysis of Elan files in Matlab," in Workshop on Multimodal Corpora at Int. Conf. for Language Resources and Evaluation (LREC 2010), Malta, 2010.

[19] G. Navarro, "A guided tour to approximate string matching," ACM Computing Surveys, vol. 33, no. 1, pp. 31-88, Mar. 2001.

[20] G. A. Fink, Markov Models for Pattern Recognition. Springer-Verlag Berlin Heidelberg, 2008.

[21] M. Bennewitz, W. Burgard, G. Cielniak, and S. Thrun, "Learning motion patterns of people for compliant robot motion," Internationl Journal of Robotics Research, vol. 24, pp. 31-48, 2005.

[22] P. Holthaus, I. Lütkebohle, M. Hanheide, and S. Wachsmuth, "Can I Help You? A Spatial Attention System for a Receptionist Robot," in Proc. Int. Conf. on Social Robotics, ser. Lecture Notes in Computer Science, S. Ge, H. Li, J.-J. Cabibihan, and Y. Tan, Eds. Springer Berlin / Heidelberg, 2010, vol. 6414, pp. 325-334. 